\title{
A NOVEL FLUID RESUSCITATION STRATEGY MODULATES PULMONARY TRANSCRIPTION FACTOR ACTIVATION IN A MURINE MODEL OF HEMORRHAGIC SHOCK
}

Todd W. Costantini, Jessica Deree, J.O. Martins, James G. Putnam BS, Tercio de Campos, Raul Coimbra

doi: $10.1590 / \mathrm{S} 1807-59322010000600010$

Costantini TW, DereeJ, Martins JO, Putnam JG, De Campos T, Coimbra R. A novel fluid resuscitation strategy modulates pulmonary transcription factor activation in a murine model of hemorrhagic shock. Clinics. 2010;65(6):621-8.

INTRODUCTION: Combining the hemodynamic and immune benefits of hypertonic saline with the anti-inflammatory effects of the phosphodiesterase inhibitor pentoxifylline (HSPTX) as a hemorrhagic shock resuscitation strategy reduces lung injury when compared with the effects of Ringer's lactate (RL). We hypothesized that HSPTX exerts its anti-inflammatory effects by interfering with nuclear factor kappa B/cAMP response element-binding protein (NF-kB-CREB) competition for the coactivator CREB-binding protein (CBP) in lung tissue, thus affecting pro-inflammatory mediator production.

METHODS: Male Sprague-Dawley rats underwent 60 minutes of hemorrhagic shock to reach a mean arterial blood pressure of $35 \mathrm{mmHg}$ followed by resuscitation with either RL or HSPTX (7.5\% HS + $25 \mathrm{mg} / \mathrm{kg}$ PTX). After four hours, lung samples were collected. NF- $\mathrm{kB}$ activation was assessed by measuring the levels of phosphorylated cytoplasmic inhibitor of kappa B (I-kB) and nuclear NF- $\mathrm{kB}$ p65 by western blot. NF- $\mathrm{kB}$ and CREB DNA-binding activity were measured by electrophoretic mobility shift assay (EMSA). Competition between NF- $\mathrm{kB}$ and CREB for the coactivator CBP was determined by immunoprecipitation. Interleukin-8 (IL-8) levels in the lung were measured by ELISA.

RESULTS: RL resuscitation produced significantly higher levels of lung IL-8 levels, I-kB phosphorylation, p65 phosphorylation, and NF- $\mathrm{kB}$ DNA binding compared with HSPTX. NF- $\mathrm{KB}-\mathrm{CBP}-$ binding activity was similar in both groups, whereas CREB-CBPbinding activity was significantly increased with HSPTX. CREB-DNA binding-activity increased to a greater level with HSPTX compared with RL.

DISCUSSION: HSPTX decreases lung inflammation following hemorrhagic shock compared with conventional resuscitation using RL through attenuation of NF- $\mathrm{KB}$ signaling and increased CREB-DNA binding activity. HSPTX may have therapeutic potential in the attenuation of ischemia-reperfusion injury observed after severe hemorrhagic shock.

KEYWORDS: Hypertonic saline; Pentoxifylline; NF-kB; CREB; CREB-binding protein.

\section{INTRODUCTION}

Hemorrhagic shock is a major cause of death during the initial phases of trauma. The duration of ischemia and the degree of reperfusion associated with hemorrhagic shock have been linked to the development of acute lung injury (ALI) and multi-system organ failure (MSOF). ALI and its most

Division of Trauma, Surgical Critical Care, and Burns, University of California San Diego School of Medicine - San Diego, California, USA.

E-mail: rcoimbra@ucsd.edu

Tel.: 619 543-7100

Received for publication on December 10, 2010

First review completed on January 11, 2010

Accepted for publication on March 02, 2010 severe form, acute respiratory distress syndrome (ARDS), are characterized by disruption of the pulmonary endothelial barrier, which leads to interstitial edema, reduced lung compliance, and persistent hypoxia. ${ }^{1}$ The rapid production of chemokines and cytokines, including interleukin-8 (IL-8), after an inflammatory insult such as hemorrhagic shock is preceded by and dependent on an increase in the corresponding mRNA transcripts and is a direct consequence of the initiation of pro-inflammatory gene transcription. ${ }^{2,3}$ These particular genes have been shown to contain promoter regions that can bind transcriptional factors, including nuclear factor-kappa B (NF-kB) and cAMP response element-binding protein (CREB), and modulate gene transcription in a positive or negative manner. ${ }^{4}$ 
Activity of the transcriptional factor NF- $\mathrm{BB}$ has been shown to correlate with the severity of illness in systemic inflammatory response syndrome (SIRS) patients and was significantly increased in nonsurvivors when compared with survivors of septic shock. ${ }^{5,6}$ In its inactive state, NF- $\mathrm{B}$, which is mainly composed of two subunits, p50 and p65, is retained in the cytoplasm and associated with the inhibitory protein, I- $\kappa \mathrm{B} .{ }^{7}$ In conditions such as ischemia-reperfusion and endotoxemia, I- $\kappa \mathrm{B} \alpha$, the major cytosolic inhibitor of $\mathrm{NF}-\kappa \mathrm{B}$, undergoes phosphorylation, ubiquitination, and subsequent proteolytic degradation by the $26 \mathrm{~S}$ proteosome. ${ }^{8,9}$ This process results in unmasking of the nuclear localization signal and translocation of p65 to the nucleus. Once in the nucleus, NF- $\kappa \mathrm{B}$ can bind with high affinity to the promoter regions of pro-inflammatory genes such as tumor necrosis factor alpha (TNF- $\alpha$ ), IL-1, IL-8, and intracellular adhesion molecule-1 (ICAM-1).

CREB is involved in cell proliferation, differentiation, and adaptive responses. Stimulus-induced activation of CREB can occur secondary to a variety of cellular stressors, including ischemia-reperfusion, and is mediated by phosphorylation of CREB at serine residue $133 .{ }^{10}$ The coactivator CREB-binding protein (CBP) and its paralogue p300 regulate transcription through selective interaction with individual transcription factors. The phosphorylated forms of both NF- $\mathrm{BB}$ and CREB have been demonstrated to bind to the same site in the N-terminal region of CBP (also called the KIX region). ${ }^{11}$ Given that $\mathrm{CBP}$ is present in the nucleus of cells in limiting amounts, competition between NF- $\kappa \mathrm{B}$ and CREB for CBP may represent an additional transcription regulatory mechanism.

The current clinical resuscitation regimen in patients with hemorrhagic shock necessitates the administration of substantial volumes of Ringer's lactate (RL). However, there is considerable evidence indicating that RL infusion enhances neutrophil activation and potentiates lung injury through upregulation of oxidative stress and expression of adhesion molecules. ${ }^{12-14}$ These findings have prompted the search for alternative resuscitation strategies.

The utilization of Pentoxifylline (PTX) as an adjunct to RL infusion and the concept of low-volume resuscitative fluids such as hypertonic saline (HS) have been extensively studied. Both strategies are capable of reducing the endorgan injury observed with RL through the attenuation of bacterial translocation, ICAM-1 expression, neutrophil sequestration, and oxidative bursts. ${ }^{15-19}$

With this knowledge, our laboratory has proposed use of both HS and PTX (HSPTX) as a low-volume antiinflammatory resuscitative fluid. In an animal model of hemorrhagic shock, we previously showed that resuscitation with HSPTX resulted in the attenuation of pulmonary neutrophil infiltration, TNF-a levels, IL-1 expression, and histological ALI. ${ }^{20}$ In this series of experiments, we further investigated the mechanism by which HSPTX attenuates pulmonary inflammation. We hypothesized that HSPTX reduces lung injury through inhibition of the NF- $\mathrm{BB}$ cascade and alteration of CBP transcription factor coactivation, thus affecting pro-inflammatory mediator production.

\section{METHODS AND MATERIALS}

The experiment was approved by the University of California San Diego Animal Subjects Committee and is in accordance with the guidelines established by the National Institutes of Health.

\section{Experimental Model}

Male Sprague-Dawley rats (300-400 g) were purchased from Harlan Sprague-Dawley (San Diego, CA). A 12hour light/dark cycle was instituted, and food and water were provided ad libitum. Animals were anesthetized with ketamine and xylazine by intraperitoneal injection. A right inguinal incision was performed, and the femoral artery and vein were cannulated with polyethylene catheters (PE50). The venous catheter was utilized for injection of resuscitative fluids, and the arterial catheter was used to withdraw blood and monitor the mean arterial pressure (MAP). Blood was withdrawn over a period of up to 10 minutes until a MAP of $35 \mathrm{mmHg}$ was obtained. Controlled hypotension was maintained at $35 \pm 5 \mathrm{mmHg}$ for 1 hour by withdrawal or reinfusion of blood as necessary. The body temperature of the animals was maintained at $37{ }^{\circ} \mathrm{C}$ throughout the experiment.

At the end of the shock period, animals were randomly divided into three groups according to the treatment received. sham animals $(n=5)$ underwent cannulation without shock or resuscitation and served as negative controls. RL-resuscitated animals $(\mathrm{n}=7)$ received $32 \mathrm{~mL} / \mathrm{kg}$ of racemic RL. HSPTXtreated animals $(\mathrm{n}=7)$ received $4 \mathrm{~mL} / \mathrm{kg}$ of $7.5 \% \mathrm{NaCl}+25$ $\mathrm{mg} / \mathrm{kg}$ of PTX (Sigma, St. Louis, MO).

The RL infusion volume was calculated to yield sodium loads equivalent to those in HSPTX-treated animals. The dose of PTX was chosen based on multiple studies from our laboratory demonstrating its safety and lack of hypotension. ${ }^{20}$ At the end of volume resuscitation, the catheters were removed, the incision was closed, and the animals were returned to their cages. The animals were sacrificed via cardiac puncture after completion of shock and resuscitation, which included the one hour duration of hemorrhagic shock and the four hours following resuscitation. 


\section{Lung Procurement and Extraction of Nuclear and Cytoplasmic Proteins}

After a median sternotomy, the right lung of each animal was excised and snap-frozen in liquid nitrogen. Lung tissue samples weighing approximately $100 \mathrm{mg}$ were homogenized using a dounce homogenizer. Nuclear and cytoplasmic proteins from the lung tissue were isolated with NE-PER ${ }^{\mathrm{TM}}$ nuclear and cytoplasmic extraction reagents supplemented with 1x Halt protease inhibitor cocktail per the manufacturer's instructions (Pierce, Rockland, IL). The total protein concentration of each extract was determined using the bicinchoninic acid protein assay according to a microplate procedure (Pierce). Absorbance was measured and a standard curve for albumin was generated at $562 \mathrm{~nm}$ using a microplate reader (Molecular Devices, Sunnyvale, CA). Extracts were then aliquoted and stored at $-70{ }^{\circ} \mathrm{C}$ until they were used in subsequent experiments.

\section{NF- $\kappa B$ and I- $\kappa B$ Western Blot Analysis}

To determine the degree of I- $\mathrm{kB}$ dissociation from NF- $\kappa B$, we measured the amount of phosphorylated I- $\kappa B$ in cytoplasmic extracts. In addition, nuclear extracts were used to determine the degree of NF- $\mathrm{KB}$ phosphorylation and translocation. In separate experiments, cytoplasmic and nuclear extracts (10 $\mu \mathrm{g}$ of protein) were separated by sodium dodecyl sulfate (SDS)-polyacrylamide gradient gel electrophoresis and transferred to nitrocellulose membranes. The membranes were blocked with blocking solution, which was composed of 5\% milk (Sigma) prepared in Trisbuffered saline/Tween 20 (Fischer Scientific, Pittsburgh, PA), for 1 hour and incubated with phosphorylated I- $\kappa \mathrm{B} \alpha$ antibody (1:200 dilution; Cell Signaling, Beverly, MA) or phosphorylated NF-kB antibody (1:500; Cell Signaling), respectively, overnight at $4{ }^{\circ} \mathrm{C}$. The membranes were washed with Tris-buffered saline/Tween 20 and incubated for 1 hour at room temperature with the secondary antibody, horseradish peroxidase-linked anti-rabbit IgG (Cell Signaling), which was prepared in blocking solution (1:2000). After washing, the membrane was processed with the Pierce Supersignal West Pico Chemiluminescent Kit and exposed to X-ray film (Hyperfilm, Amersham Pharmacia, Piscataway, NJ) to permit detection of the antibody complexes.

\section{NF-KB and CREB Electrophoretic Mobility Shift Assay}

The nonradioactive LightShift Chemiluminescent Electrophoretic Mobility Shift Assay (EMSA) Kit was used to detect DNA-transcription factor interactions. The
3 ' biotin end-labeled oligonucleotide used as a probe for the NF- $\kappa$ B EMSA was a 42-bp double-stranded oligonucleotide (5'-TTGTTACAAGGGGACTTTCCGCTG GGGACTTTCCGGGAGGC-3') containing two tandomly repeated NF- $\kappa B$ binding sites (underlined). The 3' biotin end-labeled oligonucleotide used in the CREB EMSA was a 23-bp double-stranded oligonucleotide (5'-TTTTCGAGCTCTGACGTCAGAGC-3'). Specificity for each experiment was determined by a competition assay in which $200 \mathrm{M}$ excess of unlabeled double-stranded NF$\kappa \mathrm{B}$ or CREB oligonucleotide was included in the respective reaction mixture.

Nuclear extracts $(10 \mu \mathrm{g})$ were incubated with $5 \mathrm{nM}$ NF- $\kappa B$ or CREB probe $(1 \times$ binding buffer, $150 \mathrm{mmol} / \mathrm{L}$ $\mathrm{KCl}, 0.1 \mathrm{mmol} / \mathrm{L}$ EDTA, $2.5 \mathrm{mmol} / \mathrm{L}$ DTT, 0.05\% NP40, $10 \%$ glycerol, and $50 \mathrm{ng} / \mathrm{mL}$ poly[dI-dC]) and subjected to electrophoresis through a 6\% DNA retardation gel at $100 \mathrm{~V}$ for 90 minutes. Nucleic acid was electrophoretically transferred from the gel to a positively charged nylon membrane (Roche Applied Science, Indianapolis, IN) at $380 \mathrm{~mA}$ for 1 hour on ice and immediately UV cross-linked for 15 minutes using a UV transilluminator equipped with a 312-nm bulb. Streptavidin-horseradish peroxidase conjugate and the LightShift Chemiluminescent Substrate were used in a chemical reaction to detect the biotin end-labeled oligonucleotide. The nylon membranes were exposed to $\mathrm{X}$-ray film for 1-3 minutes to permit detection.

\section{CBP Immunoprecipitation}

Interactions between CBP and CREB or p65 were demonstrated with a modification of the method reported by Gerritsen and coworkers. ${ }^{21}$ Lung nuclear extracts (10 $\mu \mathrm{g})$ were suspended in $0.5 \mathrm{M}$ phosphate lysis buffer $(\mathrm{pH}$ 8) in Eppendorf tubes (Fischer Scientific) and rotated for 20 minutes at room temperature with an anti-CBP antibody (A22) (Santa Cruz Biotechnology, Santa Cruz, CA) cross-linked to protein A magnetic beads according to the manufacturer's protocol (Invitrogen, Carlsbad, CA). The beads were washed twice with phosphate lysis buffer and eluted into SDS sample buffer (Invitrogen) by boiling for 5 minutes. The samples were then submitted to electrophoresis through SDS-polyacrylamide gels and transfer to nitrocellulose membranes. The membranes were blocked with $5 \%$ milk prepared in Tris-buffered saline/ Tween 20 for 1 hour and incubated overnight at $4{ }^{\circ} \mathrm{C}$ with anti-CBP (C1) (1:1000), anti-p65 NF-kB (1:400) and antiCREB (1:1000). Washing, secondary antibody incubation, and antibody-complex detection were performed as described above in the western blot section. 


\section{Interleukin-8 Content Determination}

The concentration of IL-8 in the lung was quantitatively measured from bronchoalveolar lavage fluid (BALF) using ELISA. Immediately after animal sacrifice, the trachea was accessed via a tracheotomy. Ten $\mathrm{ml}$ of normal saline was instilled under direct vision of the lung to avoid overdistension. BALF was collected and centrifuged at $250 \mathrm{~g}$ for 10 minutes. Similar amounts of BALF were obtained for each animal. Anti-CINC antibodies were utilized to detect rat IL-8 (Peptide Institute, Osaka, Japan). The wells of a 96-well immunoplate (NUNC Brand, Rochester, NY) were coated with the capture antibody, goat anti-CINC antibody diluted 1:396 in coating buffer, and incubated overnight at $4{ }^{\circ} \mathrm{C}$. Nonspecific binding sites were blocked with a buffer composed of $5 \%$ milk in sterile PBS (Irvine Scientific, Santa Ana, CA). The wells of the plate were washed with $0.05 \%$ Tween 20 in PBS. Undiluted BALF was incubated on the plate at room temperature for 2 hours along with IL-8 standard (Peptide Institute) diluted to the range of 0-25 ng/ $\mathrm{mL}$ in blocking buffer for standard curve generation. After washing, the secondary antibody abbit anti-CINC IgG was diluted to 1:2000 in blocking buffer and dispensed onto the plate for incubation at room temperature. The horseradish peroxidase-linked goat anti-rabbit conjugate (Cell Signaling) was instilled on the plate and incubated at room temperature for 30 minutes. An Immunopure TMB Substrate Kit (Pierce) was used for detection. The reaction was stopped by the addition of a final concentration of $2 \mathrm{M} \mathrm{H}_{2} \mathrm{SO}_{4}$ after 15 minutes, and absorbance was measured at $450 \mathrm{~nm}$. Measurements for each animal were performed in duplicate.

\section{Statistical Analysis}

Data are presented as the mean percentage of RL stimulation $+/$ - the standard error of the mean (SEM). Assays were performed in duplicate when appropriate. The percentage of RL stimulation was calculated by dividing each sample mean by the mean of RL stimulation and multiplying the number by 100 . A one-way analysis of variance (ANOVA) was used to test for significant differences between experimental groups. Statistical significance was defined as a $\mathrm{p}$ value $<0.05$.

\section{RESULTS}

\section{Cytoplasmic I- $\kappa$ B Phosphorylation}

We assessed changes in cytoplasmic $I-\kappa B a$ phosphorylation as this is a proximal step in the activation and nuclear translocation of NF- $\kappa B$. Resuscitation with RL led to a fivefold increase in I- $\mathrm{kB} \alpha$ phosphorylation compared with the level detected in sham treated animals (Figure 1). HSPTX infusion resulted in a $63 \%$ reduction in I- $\mathrm{kB} \alpha$ phosphorylation when compared with RL treatment (100 vs. $37 \pm 21 ; \mathrm{p}=0.043$ ).

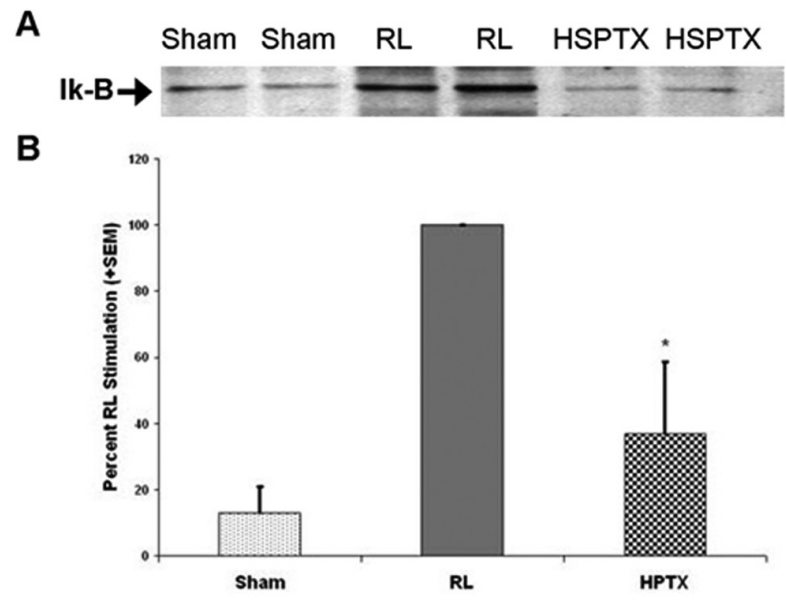

Figure 1 - Cytoplasmic I- $\kappa \mathrm{B} \alpha$ Phosphorylation. A, Representative western blot of phosphorylated cytoplasmic I- $\kappa \mathrm{B} a$ at 4 hours after hemorrhagic shock and resuscitation. The image displays two representative animals from each experimental group. B, Histogram illustrating the $63 \%$ increase in phosphorylation of I- $\kappa$ B $\alpha$ when RL, rather than HSPTX, was administered after shock. The graph presents changes in the mean density for $\mathrm{n}=5-7$ animals per group. *, p < 0.043 vs. RL.

\section{Nuclear NF-אB Phosphorylation}

To assess whether the attenuation I- $\kappa \mathrm{B} \alpha$ phosphorylation observed with HSPTX after hemorrhagic shock affected nuclear translocation of NF- $\mathrm{BB}$, we analyzed the relative amounts of phosphorylated p65 NF-kB in lung nuclear extracts. As expected, RL-treated animals had a higher degree of translocation over that of the sham group (Figure 2). The HSPTX group demonstrated a $49 \%$ decline in NF$\kappa \mathrm{B}$ nuclear transfer in comparison with their RL-treated counterparts $(51 \pm 3$ vs. $100 ; \mathrm{p}<0.01)$.

\section{NF-KB-DNA Binding Activity}

The binding of NF- $\mathrm{kB}$ to specific DNA promoter regions results in the initiation of transcription and the induction of pro-inflammatory gene expression associated with reperfusion injury. The association of p65 NF- $\mathrm{kB}$ with DNA was roughly equivalent in the sham and HSPTX groups (54 \pm 5 vs. $30 \pm 19$ ) (Figure 3). Post-shock resuscitation with RL produced a marked increase in binding over that of HSPTX (100 vs. $30 \pm 19 ; \mathrm{p}=0.01)$. These findings correlate with the relative degree of phosphorylated nuclear p65 NF-kB observed in the previous experiment. 
A

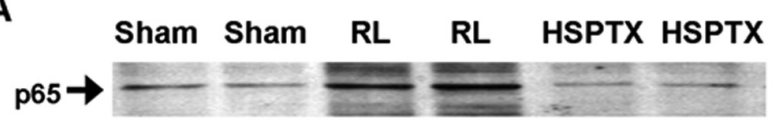

B

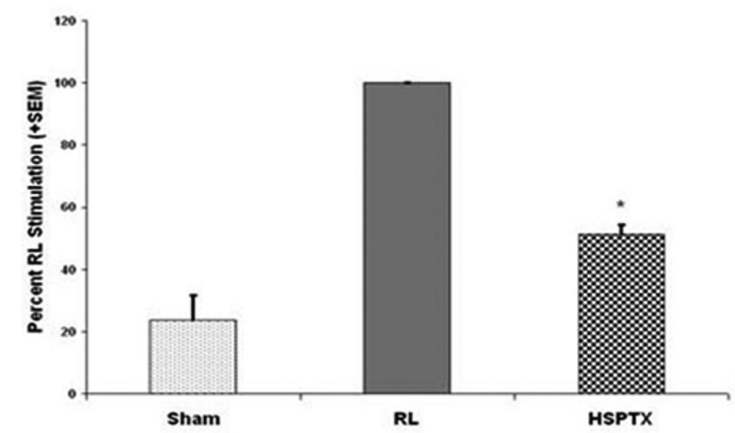

Figure 2 - Nuclear p65 NF-кB Phosphorylation. A, Western blot of phosphorylated p65 NF- $\mathrm{kB}$ in pulmonary nuclear extracts after hemorrhagic shock and resuscitation. The image displays two representative animals from each experimental group. B, RL infusion markedly enhanced nuclear NF-kB phosphorylation. A 49\% attenuation in nuclear p65 phosphorylation was seen when HSPTX was utilized for treatment after shock. The graph presents changes in the mean pixel density for $\mathrm{n}=5-7$ animals per group. *, $\mathrm{p}<0.01$ vs. $\mathrm{RL}$

A

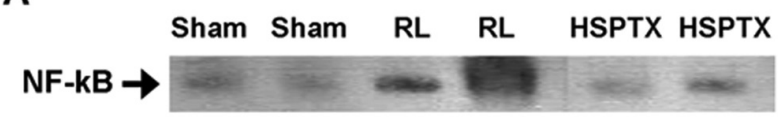

B

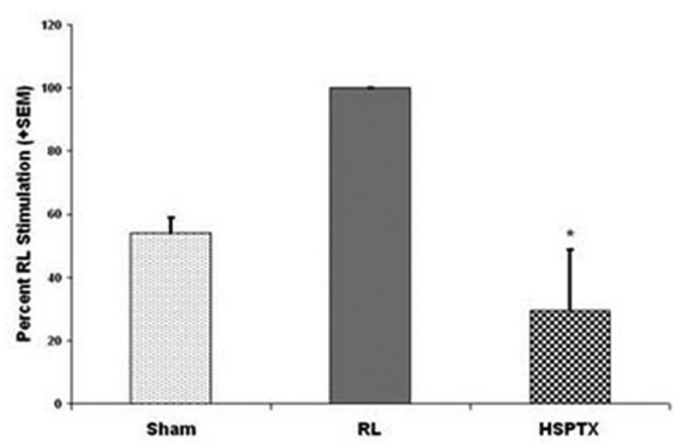

Figure 3 - NF-кB-DNA Binding Activity after Hemorrhagic Shock and Resuscitation. A, Representative EMSA illustrating NF- $\kappa$ B binding to DNA at 4 hours after post-shock resuscitation. The image displays two representative animals from each experimental group. B, Histogram demonstrating the marked upregulation of NF- $\mathrm{BB}-\mathrm{DNA}$ binding activity associated with RL treatment. The graph presents changes in mean pixel density for $\mathrm{n}=5-7$ animals per group. HSPTX treatment attenuated this response by $70 \%$ and resulted in binding levels similar to those detected in the sham group. *, $\mathrm{p}=0.01 \mathrm{vs}$. RL.

\section{CBP Interactions with NF- $\kappa B$ and CREB}

To determine whether coactivator affinity for the transcription factors associated with inflammation was affected by the type of fluid administered after hemorrhagic shock, we evaluated the relative increases in CBP binding to NF- $\kappa B$ and CREB after RL and HSPTX resuscitation. Both RL and HSPTX-treated animals had a similar degree of CBP-NF- $\kappa \mathrm{B}$ binding activity (10.56\% vs. $10.25 \%)$. Resuscitation with HSPTX resulted in a marked rise in the association between CBP and CREB when compared with RL treatment ( $24 \%$ vs. $4.34 \%$ ) (Figure 4 ). These data indicate that RL given after hemorrhagic shock favors co-activation of NF- $\mathrm{kB}$ and subsequent pro-inflammatory mediator synthesis. With HSPTX resuscitation, the association between CREB and CBP outweighs that demonstrated with NF-kB.

A $\begin{array}{llll}1 & 1 & 2 & 2\end{array}$

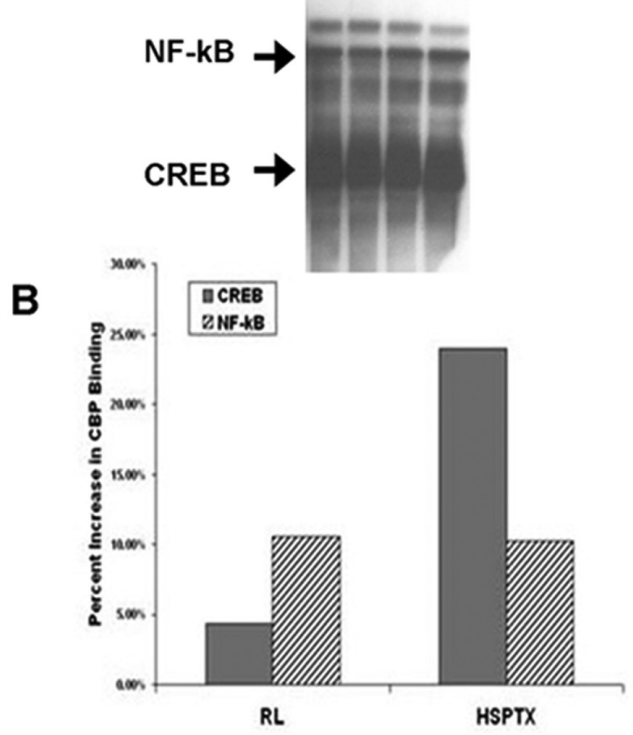

Figure 4 - Alterations in CBP Binding Activity After Resuscitation. A, Representative immunoprecipitation blot of animals resuscitated with either RL (1) or HSPTX (2). The image displays two representative animals from each experimental group. B, Graph demonstrating the percent increases in NF- $\mathrm{BB}$ and CREB CBP-binding activity after resuscitation with RL or HSPTX. Treatment with RL resulted in a greater proportion of NF- $\kappa B$ coactivation, while CBP-CREB binding activity was shown to predominate with HSPTX treatment. The graph presents changes in mean pixel density for $\mathrm{n}=5-7$ animals per group.

\section{CREB-DNA Binding Activity}

Given that an increased association between CBP and CREB was evident with HSPTX, we sought to determine whether CREB-DNA binding was upregulated. HSPTXtreated animals demonstrated a $93 \%$ increase in the association of CREB and DNA over their RL counterparts $(193 \pm 11$ vs. $100 ; p=0.001)$ (Figure 5). Both sham and RLtreated animals had similar levels of CREB DNA-binding activity (110 \pm 6 vs. 100).

\section{Interleukin-8 Concentration}

Synthesis of the pro-inflammatory mediator IL-8 is partly influenced by activation of the transcription factor NF- $\mathrm{kB}$. IL- 
A Sham Sham RL RL HSPTX HSPTX CREB

B

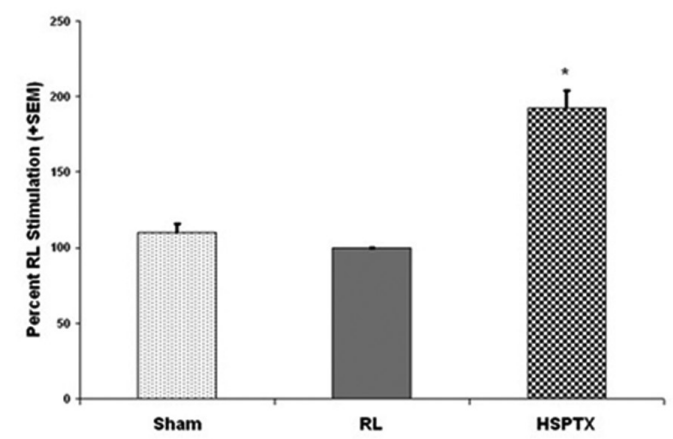

Figure 5 - CREB-DNA Binding Activity After Hemorrhagic Shock and Resuscitation. A, Representative EMSA illustrating CREB DNA-binding activity at 4 hours after post-shock resuscitation. The image displays two representative animals from each experimental group. B, Histogram presenting changes in mean pixel density for $n=5-7$ animals per group compared with RL-treated animals. Enhanced CREB-DNA binding activity was observed in animals receiving HSPTX infusion. RL-treated animals had CREB-DNA association levels similar to the sham group. *, $\mathrm{p}=0.001 \mathrm{vs.}$ RL.

8 regulates chemotaxis, directional neutrophil migration, and neutrophil activation in the lung during ischemia-reperfusion injury. Resuscitation with RL caused a significant increase in the BALF IL- 8 concentration at 4 hours compared with sham animals ( $100 \%$ vs. $38 \% \pm 6 ; p<0.0001)$ (Figure 6). HSPTX resuscitation led to a $55 \%$ decrease in IL-8 levels when compared with their RL counterparts $(45 \% \pm 9$ vs. $100 ; p<$ 0.0001 ). This reduction in cytokine expression correlates with the decline in NF- $\mathrm{KB}$ activity, coactivation, and DNA binding activity observed with HSPTX.

\section{DISCUSSION}

Hemorrhagic shock is the most prominent cause of patient morbidity and mortality after severe trauma. In

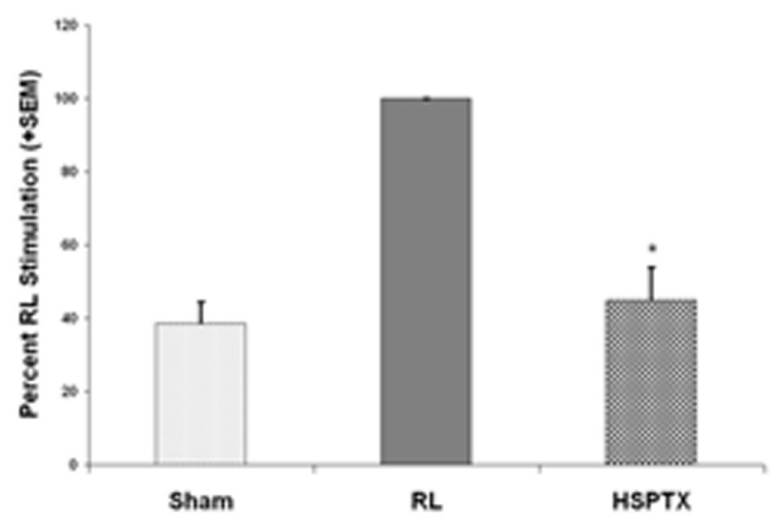

Figure 6 - Resuscitation-induced Lung Interleukin-8 Levels. Animals resuscitated with RL had increased concentrations of IL- 8 in the lung. Resuscitation with HSPTX led to a 55\% attenuation of IL- 8 . The graph presents mean BALF IL-8 levels of all animals studied in each group. *, $p<0.0001$ vs. RL. contrast to those who die early as a result of uncontrolled bleeding, patients who survive the initial period of shock and resuscitation may develop an uncontrolled systemic inflammatory response that culminates with end-organ injury. Cytokines and reactive oxygen species produced during the reperfusion period are responsible for neutrophil and monocyte/macrophage activation, which, if unregulated, results in the development of ALI and ARDS. ${ }^{22}$

PTX, a methylxanthine derivative and nonspecific phosphodiesterase inhibitor, has classically been used for the treatment of intermittent claudication due to its hemorheologic properties. ${ }^{23}$ More recently, PTX has been shown to yield beneficial effects on the inflammatory cascade by increasing cAMP and subsequently reducing TNF- $\alpha$ levels. ${ }^{24}$ Our laboratory and others have extensively studied PTX as an immunomodulator in hemorrhagic shock. In animal models, the administration of PTX after hemorrhage improves tissue oxygenation, intestinal blood flow, and animal survival. ${ }^{25-27}$ PTX alone is not a volume expander and cannot be used as the sole treatment for shock. Therefore, it must be given as an adjunct therapy with the appropriate volume resuscitation.

The concept of small-volume resuscitation consisting of $4 \mathrm{~mL} / \mathrm{kg}$ of $7.5 \% \mathrm{NaCl}$ (HS) in the setting of hemorrhagic shock has been a topic of recent research. HS not only offers the advantages of more rapid administration and almost instantaneous hemodynamic stability, it also downregulates neutrophil activation and organ injury in comparison with RL. ${ }^{14-16,18}$ With these findings in mind, our laboratory has utilized HSPTX as a combined fluid for post-shock resuscitation and has demonstrated a reduction in endorgan injury and pro-inflammatory mediator production in comparison with RL. ${ }^{19}$ We have previously shown that treatment with HSPTX decreases histological lung injury using an animal model of hemorrhagic shock..$^{20}$ In this series of experiments, we further investigated the effects of resuscitation with HSPTX on pulmonary inflammation in an animal model of hemorrhagic shock.

In this study, we chose to study changes in lung inflammation at the 4-hour time point based on our previous studies using this animal model of hemorrhagic shock. HSPTX infusion resulted in a significant reduction in pulmonary IL-8 levels when compared with RL administration. Molecular studies examining IL- 8 gene activation have shown that the majority of IL- 8 gene transcription is mediated by activation of NF- $\mathrm{kB}$ and that inhibition of NF- $\mathrm{B}$ expressionusing antisense oligonucleotide inhibits IL-8 production in vitro ${ }^{8,29}$ Therefore, the attenuation of IL- 8 expression observed in this study is most likely due to downregulation of the NF-kB cascade induced by HSPTX therapy. 
One of the key steps involved in NF-kB activation is phosphorylation of the inhibitory protein I- $\kappa \mathrm{B} \alpha$, which allows for subsequent $\mathrm{I}-\mathrm{\kappa B}$ degradation and NF- $\mathrm{kB}$ activation. ${ }^{30}$ In vivo inhibition of $\mathrm{I}-\mathrm{\kappa B} \alpha$ degradation has been shown to suppress lung inflammation in septic rats. ${ }^{31}$ In this study, we observed marked attenuation in I- $\mathrm{kB} \alpha$ phosphorylation in animals resuscitated with HSPTX after hemorrhagic shock, indicating a mechanism by which HSPTX exerts its anti-inflammatory effects.

Multiple studies have demonstrated that agents that increase cAMP, such as PTX, cause activation of protein kinase A (PKA) and lead to inhibition of NF-kB-dependent pro-inflammatory gene expression..$^{32-34}$ Haddad et al. reported results that are consistent with our study, showing the effects of PTX on I- $\kappa \mathrm{B}$ and NF- $\kappa \mathrm{B}$ in pulmonary epithelial cells. ${ }^{35}$ For NF- $\mathrm{kB}$ to recruit the transcriptional apparatus and stimulate gene expression, it must first undergo nuclear translocation and p65 subunit modification. Once in the nucleus, the p65 subunit is phosphorylated at serine 276, which enhances its ability to recruit CBP and p300 and subsequently bind to DNA. ${ }^{36,37}$ Here, HSPTXresuscitated animals displayed a marked reduction in

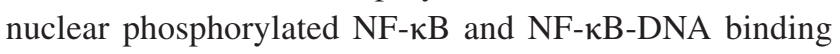
activity when compared with their RL-treated counterparts. Therefore, the attenuation of NF-kB activity exhibited with HSPTX is a consequence of the inhibition of phosphorylation of both I- $\mathrm{kB}$ in the cytoplasm and p65 in the nucleus.

In contrast to NF- $\kappa \mathrm{B}, \mathrm{CREB}-\mathrm{DNA}$ binding activity was significantly upregulated when HSPTX was utilized for post-shock resuscitation. Phosphorylation of CREB at serine 133 is primarily the result of a Protein Kinase A (PKA)dependent mechanism and is required for CREB activation. ${ }^{10}$ Therefore, the elevation in cAMP that occurs with HSPTX resuscitation downregulates $\mathrm{NF}-\kappa \mathrm{B}$ expression while increasing the activity of the anti-inflammatory transcription factor CREB, thus modulating pro-inflammatory mediator synthesis and reducing lung inflammation. This concept is supported by our previous studies demonstrating attenuation of TNF- $\alpha$ synthesis with HSPTX both in vitro and in vivo $0^{20,38}$
Given that the interaction between both CREB and NF$\kappa \mathrm{B}$ occurs through the same region of CBP (the KIX region), the competition between transcription factors for a finite amount of coactivator has the potential to be an additional transcription regulatory mechanism. ${ }^{21,39}$ In this study, the association between $\mathrm{CBP}$ and NF- $\mathrm{kB}$ predominated with standard RL resuscitation. In contrast, CREB-CBP binding was favored with HSPTX resuscitation, potentially accounting for the difference in pro-inflammatory mediator synthesis observed with different resuscitation modalities. These data are supported by the findings of Shenkar et al., who similarly demonstrated that inhibition of xanthine oxidase and production of reactive oxygen species in mice before hemorrhage reduced interactions between $\mathrm{p} 65$ and $\mathrm{CBP}$, while CREB-CBP associations were significantly increased. ${ }^{11}$

We did not choose to use an HS-only control group, which may be viewed as a limitation of this study. We and others have extensively studied the immunomodulatory effects of hypertonic saline alone. The goal of this study was to combine a small-volume immunomodulatory resuscitation fluid (HS) with an anti-inflammatory drug (PTX). This strategy combines anti-inflammatory strategies and provides a vehicle for the delivery of PTX. The benefits of this combination in a small volume of fluid could be ideal for pre-hospital fluid therapy or resuscitation in the field.

\section{CONCLUSION}

The use of HSPTX for fluid resuscitation after hemorrhagic shock may reduce the incidence of lung inflammation through attenuation of I-kB phosphorylation, $\mathrm{NF}-\kappa \mathrm{B}$ p65 nuclear translocation, CBP coactivation, and NF- $\mathrm{kB}-\mathrm{DNA}$ binding activity. These alterations, coupled with increased CREB activity, led to diminished pro-inflammatory mediator production, which may have contributed to a decrease in tissue injury when compared with RL, the current standard resuscitation fluid. Therefore, this novel resuscitation strategy may have therapeutic potential in the attenuation of ischemia-reperfusion injury observed after severe hemorrhagic shock.

\section{REFERENCES}

1. Aldridge AJ. Role of the Neutrophil in Septic Shock and the adult respiratory distress syndrome. Eur J Surg. 2002;168:204-14.

2. Bazzoni F, Cassatella MA, Laudanna C, Rossi F. Phagocytosis of opsonized yeast induces tumor necrosis factor- $\alpha$ mRNA accumulation and protein release by human polymorphonuclear leukocytes. J Leukoc Biol. 1991;50:223-8.
3. Strieter RM, Kasahara K, Allen RM, Standiford TJ, Rolfe MW, Becker FS, et al. Cytokine-induced neutrophil-derived interleukin-8. Am J Path. 1992;141:397-407.

4. Sha WC. Regulation of immune responses by NF- $\kappa \mathrm{B} / \mathrm{Rel}$ transcription factors. J Exp Med. 1998;187:143-6. 
5. Nakamori Y, Koh T, Ogura H, Tanaka H, Fujimi S, Kasai K, et al. Enhanced expression of intranuclear $\mathrm{NF}-\kappa \mathrm{B}$ in primed polymorphonuclear leukocytes in systemic inflammatory response syndrome patients. J Trauma. 2003;54:253-60.

6. Arnalich F, Garcia-Palomero E, Lopez J, Jimenez M, Madero R, Renart $\mathrm{J}$, et al. Predictive value of nuclear factor $\kappa \mathrm{B}$ activity and plasma cytokine levels in patients with sepsis. Infect Immun. 2000;68:1942-5.

7. Siebenlist U, Franzoso G, and Brown K. Structure, regulation, and function of NF-кB. Annu Rev Cell Biol. 1994;10:405-55.

8. Baldwin AS. The NF- $\kappa B$ and I- $\kappa$ B proteins; new discoveries and insights. Annu Rev Immunol. 1996;14:649-81.

9. Tran K, Merika M, Thanos D. Distinct functional properties of IkappaB alpha and IkappaB beta. Mol Cell Biol 1997;17:5386-99.

10. Shaywitz AJ, Greenberg ME. CREB: a stimulus-induced transcription factor activated by a diverse array of extracellular signals. Annu Rev Biochem. 1999;68:821-61.

11. Shenkar R, Yum K, Arcaroli J, Kupfner J, Abraham E. Interactions between $\mathrm{CBP}, \mathrm{NF}-\kappa \mathrm{B}$, and CREB in the lungs after hemorrhage and endotoxemia. Am J Physiol Lung Cell Mol Physiol. 2001;281:L418-L26.

12. Savage SA, Fitzpatrick CM, Kashyap VS, Clouse WD, Kerby JD. Endothelial dysfunction after lactated ringer's solution resuscitation for hemorrhagic shock. J Trauma. 2005;59:284-90.

13. Koustova E, Stanton K, GushchinV, Alam HB, Stegalkina S, Rhee PM Effects of lactated ringer's solutions on human leukocytes. J Trauma. 2002;52:872-8.

14. Alam HB, Stanton K, Koustova E, Burris D, Rich N, Rhee PM. Effect of different resuscitation strategies on neutrophil activation in a swine model of hemorrhagic shock. Resuscitation. 2004;60:91-9.

15. Yada-Langui MM, Coimbra R, Lancellotti C, Mimica I, Garcia C, Correia N Jr, et al. Hypertonic saline and pentoxifylline prevent lung injury and bacterial translocation after hemorrhagic shock. Shock. 2000;14:594-8.

16. Rizoli SB, Kapus A, Fan J, Li YH, Marshall JC, Rotstein OD. Immunomodulatory effects of hypertonic resuscitation on the development of lung inflammation following hemorrhagic shock. J Immunol. 1998;161:6288-96.

17. Coimbra R, Melbostad H, Loomis W, Porcides R, Wolf P, Tobar M, et al. LPS-induced acute lung injury is attenuated by phosphodiesterase inhibition: effects on pro-inflammatory mediators, metalloproteinases, NF-кB, and ICAM-1 expression. J Trauma. 2006;60:115-25.

18. Rizoli SB, Rhind SG, Shek PN, Inaba K, Filips D, Tien H, et al. The immunomodulatory effects of hypertonic saline resuscitation in patients sustaining traumatic hemorrhagic shock. Ann Surg. 2006;243:47-57.

19. Deree J, Martins JO, Leedom A, Lamon A, Putnam J, et al. Hypertonic Saline and Pentoxifylline reduces hemorrhagic shock resuscitationinduced pulmonary inflammation through attenuation of neutrophil degranulation and proinflammatory mediator synthesis. J Trauma. 2007;62:104-11.

20. Coimbra R, Porcides R, Loomis W, Melbostad H, Lall R, Deree J, et al. HSPTX protects against hemorrhagic shock resuscitation-induced tissue injury: an attractive alternative to ringer's lactate. J Trauma. 2006;60:41-51.

21. Gerritsen ME, Williams AJ, Neish AS, Moore S, Shi Y, Collins T. CREBbinding protein/p300 are transcriptional coactivators of p65. Proc Natl Acad Sci. 1997;94:2927-32.

22. Lee WL and Downey GP. Neutrophil activation and acute lung injury. Curr Opin Crit Care. 2001;7:1-7.
23. Porter JM, Cutler BS, Lee BY, Reich T, Reichle FA, Scogin JT, et al. Pentoxifylline efficacy in the treatment of intermittent claudication: Multicenter double-blind trial with objective assessment of chronic occlusive arterial disease patients. Am Heart J. 1982;104:66-72.

24. Coimbra R, Melbostad H, Hoyt DB. Effects of phosphodiesterase inhibition on the inflammatory response following shock: role of pentoxifylline. J Trauma. 2004;56:442-9.

25. Waxman K. Shock: ischemia, reperfusion, and inflammation. New Horiz. 1996;4:153-60.

26. Flynn WJ, Cryer HG, Garrison RN. Pentoxifylline restores intestinal microvascular blood flow during resuscitated hemorrhagic shock. Surgery. 1991;110:350-6.

27. Barroso-Aranda $\mathrm{J}$ and Schmid-Schonbein GW. Pentoxifylline pretreatment decreases the pool of circulating activated neutrophils, invivo adhesion to endothelium, and improves survival from hemorrhagic shock. Biorheology. 1990;27:401-18.

28. Mukaida N, Okamoto S, Ishikawa Y, Matsushima K. Molecular mechanism of interleukin-8 gene expression. J Leuk Biol. 1994;56:5548.

29. Yoshida S, Ono M, Shono T, Izumo H, Ishibashi T, Suzuki H, et al. Involvement of interleukin-8, vascular endothelial growth factor, and basic fibroblast growth factor in tumor necrosis factor alpha-dependent angiogenesis. Mol Cell Biol. 1997;17:4015-23.

30. Renard P, Percherancier Y, Kroll M, Thomas D, Virelizier J, Arenzana-Seisdedos $\mathrm{F}$, et al. Inducible $\mathrm{NF}-\kappa \mathrm{B}$ activation is permitted by simultaneous degradation of nuclear I $\mathrm{B} \alpha$. J Biol Chem. 2000;275:15193-9.

31. Lancaster LH, Christman JW, Blackwell TR, Koay MA, Blackwell TS. Suppression of lung inflammation in rats by prevention of NF- $\kappa B$ activation in the liver. Inflammation. 2001;25:25-31.

32. Coimbra R, Melbostad H, Loomis W, Tobar M, Hoyt DB. Phosphodiesterase inhibition decreases nuclear factor- $\mathrm{kB}$ activation and shifts the cytokine response toward anti-inflammatory activity in acute endotoxemia. J Trauma. 2005;59:575-82.

33. Ollivier V, Parry GC, Cobb RR, de Prost D, Mackman N. Elevated cyclic

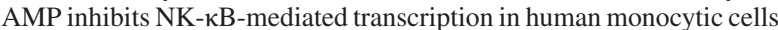
and endothelial cells. J Biol Chem. 1996;271:20828-35.

34. Minguet S, Huber M, Rosenkranz L, Schamel WA, Reth M, Brummer $\mathrm{T}$. Adenosine and cAMP are potent inhibitors of the NF- $\kappa \mathrm{B}$ pathway downstream on immunoreceptors. Eur J Immunol. 2005;35:31-41.

35. Haddad JJ, Land SC, Tarnow-Mordi WO, Zembala M, Kowalczyk D, Lauterbach R. Immunopharmacological potential of selective phosphodiesterase inhibition. II. Evidence for the involvement of an inhibitory $-\kappa \mathrm{B} /$ nuclear factor- $\kappa \mathrm{B}$-sensitive pathway in alveolar epithelial cells. J Pharmacol Exp Ther. 2002;300:567-76.

36. Zhong H, May MJ, Jimi E, Ghosh S. The phosphorylation status of nuclear NF-kappa B determines its association with CBP/p300 or HDAC-1. Mol Cell. 2002;9:625-36.

37. Naumann $\mathrm{M}$ and Scheidereit C. Activation of NF- $\kappa \mathrm{B}$ in vivo is regulated by multiple phosphorylations. EMBO J. 1994;13:4597-607.

38. Coimbra R, Loomis W, Melbostad H, Tobar M, Porcides RD, Lall $\mathrm{R}$, Holbrook et al. Role of hypertonic saline and pentoxifylline on neutrophil activation and tumor necrosis factor- $\alpha$ synthesis: a novel resuscitation strategy. J Trauma. 2005;59:257-65.

39. Shenkar R and Abraham E. Mechanisms of lung neutrophil activation after hemorrhage or endotoxemia: roles of reactive oxygen intermediates, NF- $\kappa \mathrm{B}$, and cyclic AMP response element binding protein. J Immunol. 1999; 163:954-62. 